Ann. Zootech., I978, 27 (1), 83-.93

\title{
Influence de la température ambiante sur les cinétiques des températures corporelles et confort thermique des porcelets nouveau-nés
}

\author{
A. DUSSUEL et P. BERBIGIER \\ Station de Bioclimatologie, \\ Centre national de Recherches agronomiques, I.N.R.A., \\ 7800 o Versailles (France)
}

\section{Résumé}

La température superficielle de peau (Ts) mesurée par radiothermonétrie infra-rouge, ainsi que la température rectale ( $\mathrm{Tr}$ ) de 33 porcelets de race Large White, sont enregistrées en continu pendant la première heure de vie de l'animal. On a étudié les variations des températures corporelles et l'évolution des pertes de chaleur des porcelets pour des températures ambiantes (Ta) comprises entre 23 et $40^{\circ} \mathrm{C}$.

La régulation initiale de température ( ${ }^{\text {re }}$ heure de vie) semble se situer principalement au niveau superficiel, sauf pour les animaux les plus légers ( $P \leqslant 850 \mathrm{~g}$ ) qui ont des difficultés à maintenir leur température de surface (I's) constante. Cette régulation a probablement une origine musculaire (frisson).

Ce mécanisme de régulation superficielle chez le nouveau-né est très différent de celui du porc homéotherme plus âgé (régulation centrale); cependant la forme des courbes de confort thermique (production de chaleur en fonction de Ta) est la même.

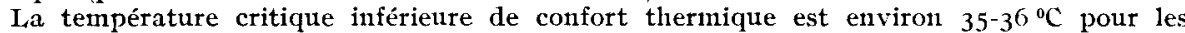
animaux de poids supérieur à $85^{\circ} \mathrm{g}$. Celle-ci peut être déterminée avec précision par la mesure de la température de surface (Ts) : en effet, on observe vers $35-36^{\circ} \mathrm{C}$, une augmentation importante de 'Ts, caractéristique du confort thermique.

\section{I. - Introduction}

Le porcelet nouveau-né est très sensible au froid. En effet ses réserves énergétiques sont faibles par rapport à d'autres espèces (WIDDowson, I950), son isolation thermique est mauvaise (pelage peu dense, absence de graisse sous-cutanée); de plus, il n'acquiert que progressivement son homéothermie. Cependant certains mécanismes sont constatés très tôt : frisson dès la naissance, érection du pelage à partir du 6-7 jour (BUTCHBAKER et SHANkLIN, 1965).

La survie et la croissance du porcelet nouveau-né dépendent fortement des 
conditions de confort thermique. En effet, on enregistre la perte moyenne de I3 p. cent des animaux nés-vivants pendant les trois premiers jours de vie (KERNKAMP, I965; NIELSEN et al., I974; ENGLISH, I976), le plus souvent par hypoglycémie fatale et écrasement par la mère.

La chute de la température rectale (Mount, I959; DE LA PORTE DEs VAUX et Aumaitre, I967; Kovacs, RafaI et SallaI, I967), suivie de la mobilisation des réserves énergétiques, principalement des glucides (ELNEIL et MCCANCE, I965; DE LA PORTE DES VAUX et AUMAITRE, I967), est bien connue, mais les études concernant la thermorégulation durant les premiers instants suivant la naissance sont plus rares ou inexistantes, sans doute par suite de l'inadaptation des moyens d'investigation classique (calorimètres et chambres respiratoires) à l'étude d'un phénomène variant rapidement au cours du temps. Par contre, des mesures simples et instantanées de température cutanée sont parfaitement adaptées à l'étude d'un tel phénomène.

Dans l'expérience présente, nous avons cherché à examiner les variations des températures corporelles des animaux en fonction de la température ambiante, et à déterminer le confort thermique pendant la première heure de vie.

\section{II. - Matériel et méthodes}

Les expériences ont porté sur 33 porcelets (I porcelet par portée) de race Large White. On a choisi le poids des animaux de façon à obtenir trois classes de poids d'effectif comparable : $600<\mathrm{P} \leqslant 850 \mathrm{~g}, 850 \mathrm{~g}<\mathrm{P} \leqslant \mathrm{I}$ I $00 \mathrm{~g}, \mathrm{P}>\mathrm{I}$ I $00 \mathrm{~g}$.

Dès la naissance, la membrane amniotique est retirée, le porcelet est pesé, puis placé dans une enceinte thermostatée $\left(0,3 \mathrm{~m}^{3}\right)$ où il est maintenu suspendu par des sangles minces selon la technique de STомвAUGH et al., I973. La température ambiante dans l'enceinte peut varier entre 20 et $40^{\circ} \mathrm{C}$ suivant le traitement; les parois sont à la température de l'air intérieur.

Les températures rectale $(\mathrm{Tr})$ et ambiante ( $\mathrm{Ta}$ ) sont mesurées à l'aide de thermocouples cuivre-constantan, la température de surface (Ts) est mesurée sur le dos de l'animal à l'aide d'un radiothermomètre (PERRIER, I970). L'humidité relative de l'air est déterminée avec une sonde au chlorure de lithium, la vitesse de l'air (mesurée avant les expériences) avec un anémomètre à fil chaud (Chiapale, I970).

Les températures rectale (Tr) et superficielle (Ts) des porcelets sont enregistrées en continu pendant l'heure suivant la naissance. L'humidité relative de l'air est mesurée à $10 \mathrm{~cm}$ environ au-dessus de l'animal, hors de sa couche limite, de $5 \mathrm{mn}$ en $5 \mathrm{mn}$.

\section{III. - Résultats}

\section{A. - Vitesse et humidité de l'air dans l'enceinte}

La vitesse de l'air à l'intérieur de l'encente, très constante, est de $6 \mathrm{~cm} / \mathrm{s}$. L'humidité relative de l'air ambiant n'est pas contrôlée, mais la pression partielle de vapeur d'eau demeure faible et pratiquement constante $(8,5 \mathrm{~mm}$ de mercure $)$ en-dessous d'une ten:pérature ambiante de $35^{\circ} \mathrm{C}$; au-delà, elle s'élève assez fortement ( $16 \mathrm{~mm}$ de mercure à $39^{\circ} \mathrm{C}$ ). 


\section{B. - Influence de la température ambiante et du poids de l'animal sur les températures superficielle et rectale}

\section{I. - Température superficielle (Ts)}

La figure Ia représente les variations de la température superficielle (Ts), prise au temps $t$ où la température rectale ( $\mathrm{Tr}$ ) est minimum (valeurs correspondant à un équilibre thermogenèse-thermolyse), en fonction de la température ambiante (Ta). Le temps mis pour atteindre ce minimum présente une grande variabilité suivant les animaux : de $\mathrm{I}_{5} \mathrm{mn}$ à plus d'une heure après la naissance.

On constate (fig. $\mathrm{I} a$ ) :

- qu'en-dessous de $36^{\circ} \mathrm{C}$, malgré une certaine variabilité individuelle, la température de surface dépend peu de la température ambiante;

- que cependant, pour les animaux les plus légers $(600<\mathrm{P} \leqslant 850 \mathrm{~g}$ environ), la température de surface (Ts) est toujours inférieure à celle des animaux plus lourds $(\mathrm{P}>850 \mathrm{~g})$, à température ambiante égale; l'écart diminue lorsque la température ambiante augmente;

- qu'au-dessus de $36^{\circ} \mathrm{C}$, Ts augmente très vite avec $\mathrm{Ta}$, du moins pour les animaux les plus lourds $(\mathrm{P}>850 \mathrm{~g})$.

Une analyse de covariance, limitée aux températures ambiantes comprises entre 23 et $36^{\circ} \mathrm{C}$, permet de préciser l'effet du poids. On a repris les trois classes de poids définies plus haut (cf. $§$ II). On montre qu'on peut confondre les valeurs obtenues par les animaux représentant les deux classes de poids supérieures à $850 \mathrm{~g}$ (pas de différences significatives).

La comparaison des droites de régression correspondant aux deux classes $(600<\mathrm{P} \leqslant 850 \mathrm{~g}$ et $\mathrm{P}>850 \mathrm{~g}:$ tabl. $\mathrm{r} a$ et fig. $\mathrm{I} a)$ montre que les animaux les plus lourds ont une température de surface (Ts) significativement supérieure à celle des animaux plus légers; cependant, la figure i $a$ semble montrer que lorsque la température ambiante ( $\mathrm{Ta}$ ) augmente, l'écart diminue. L'analyse statistique ne permet pas de conclure définitivement sur ce point : le test d'horizontalité des droites et la comparaison de leurs pentes semblent en contradiction. Mais, étant donné le faible nombre d'animaux légers $(600<\mathrm{P} \leqslant 850 \mathrm{~g})$, les différences sont à la limite de signification.

I1 semble donc que, pour des températures de l'air comprises entre 23 et $36^{\circ} \mathrm{C}$, la température de surface (Ts) ne dépende pratiquement pas de Ta pour les animaux les plus lourds $(P>850 \mathrm{~g})$, alors qu'elle augmente avec $\mathrm{Ta}$ pour les plus légers ( $600<\mathrm{P} \leqslant 850 \mathrm{~g}$ ), tout en restant inférieure à celle des animaux de poids supérieur à $850 \mathrm{~g}$.

\section{2. - Température rectale}

La figure $\mathrm{i} b$ représente les valeurs minima de la température rectale ( $\mathrm{Tr}$ ) en fonction de la température ambiante ( $\mathrm{Ta}$ ).

La comparaison de cette figure avec la figure I $a$ permet de montrer :

- que, comme pour la température de surface (Ts), les animaux de poids faible $(600<P \leqslant 850 \mathrm{~g})$ ont une température rectale minimum inférieure à celle des animaux plus lourds $(\mathrm{P}>850 \mathrm{~g})$, du moins pour des températures ambiantes inférieures à $30^{\circ} \mathrm{C}$ environ; 

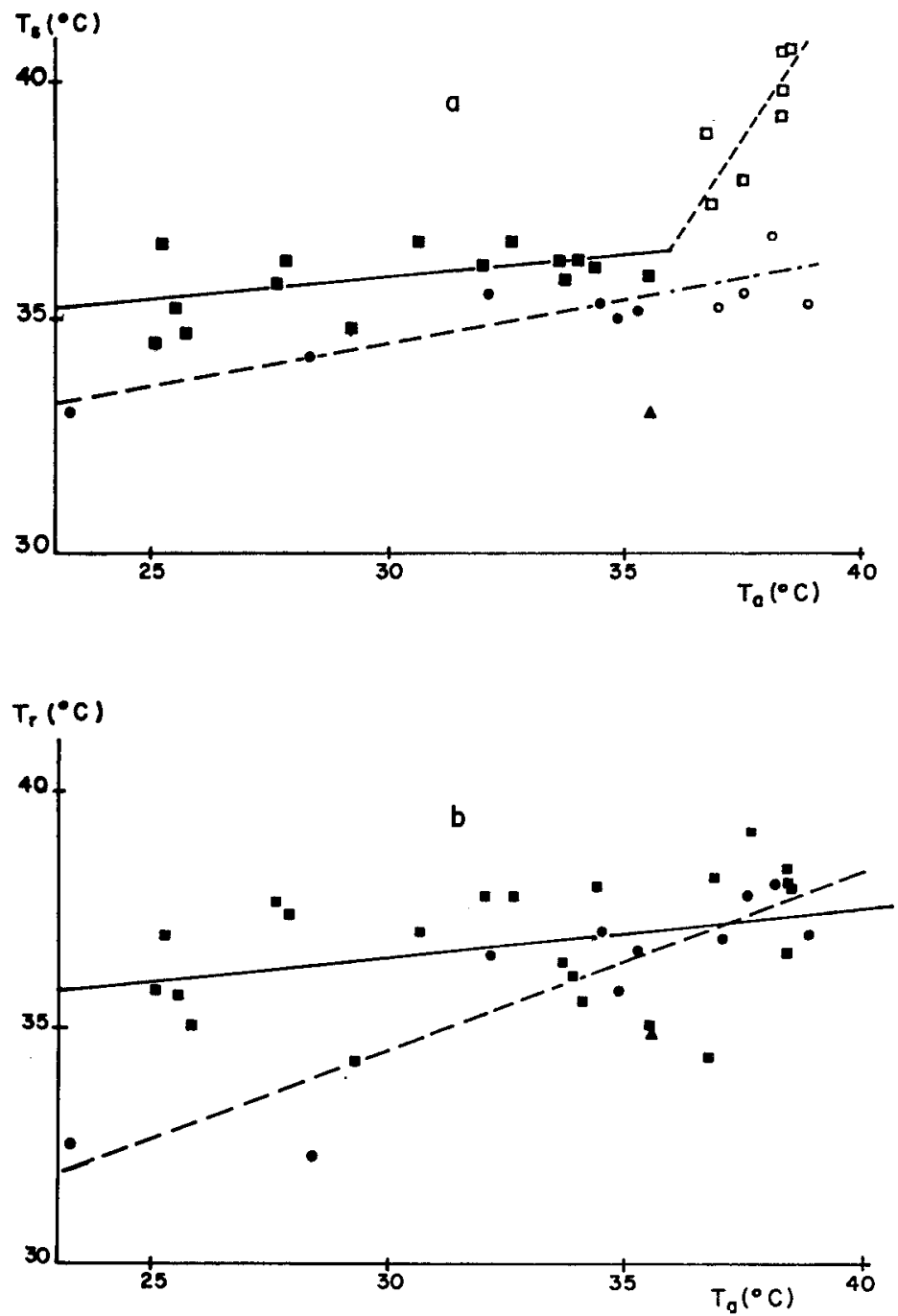

FIG. Ia. - Température de surface (TS), au temps t correspondant au minimum de température rectale $(T \gamma)$, en fonction de la température ambiante (Ta).

Surface temperature (Ts) at time $t$ wheve rectal temperatuve $(T \gamma)$ reaches its minimum value, as a function of ambient temperature (Ta).

—. $\mathrm{P}>85^{\circ} \mathrm{g}, \mathrm{Ta}<3^{\circ}{ }^{\circ} \mathrm{C} ; \quad \ldots . . . \mathrm{P}>85^{\circ} \mathrm{g} ; \mathrm{Ta} \geqslant 36^{\circ} \mathrm{C}$

- $600 \mathrm{~g}<\mathrm{P} \leqslant 85^{\circ} \mathrm{g}, \mathrm{Ta}<3^{\circ}{ }^{\circ} \mathrm{C} ;-\longrightarrow-0600 \mathrm{~g}<\mathrm{P}<85^{\circ} \mathrm{g} ; \mathrm{Ta} \geqslant 3^{\circ} \mathrm{C}$ - $\mathrm{P}=55^{\circ} \mathrm{g}$

FIG. Ib. - Température rectale (Tr), au temps $t$ où elle est minimum, en fonction de la température ambiante $(T a)$.

Rectal temperature $(T r)$ at time $t$ where it reaches its minimum value, as a function of anbient temperature (Ta).

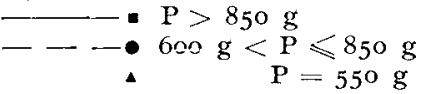




\section{TABLEAU I}

Régression de $T s(Y)$ et $\operatorname{Tr}(Y)$ sur $T a(X)$ pour les classes de poids : $600<\mathrm{P} \leqslant 850 \mathrm{~g}$ et $\mathrm{P}>850 \mathrm{~g}$

Regression of $T s(Y)$ and $\operatorname{Tr}(Y)$ on $T a(X)$ for weight classes :

Test de l'horizontalité et de comparaison des droites de régression $Y(X)$

Test of null hypothesis on regression coefficients and comparison of regression lines $Y(X)$

I $a:$ Ts $(\mathrm{Y})$ sur $\mathrm{Ta}(\mathrm{X})$

\begin{tabular}{|c|c|c|c|c|}
\hline $\begin{array}{c}\text { Classes de poids } \\
\text { Nombre } \\
\text { de couples } \\
\text { (Weight classes } \\
\text { Number } \\
\text { of couples) }\end{array}$ & $\begin{array}{c}\text { Droites de régression } \\
\text { Coefficients } \\
\text { de corrélation } \\
\text { (Regression lines } \\
\text { Correlation coefficients) }\end{array}$ & $\begin{array}{c}\text { Test } \\
\text { de l'horizontalité } \\
\text { de la droite } \\
\text { (Test } \\
\text { of null hypothesis } \\
\text { on regression } \\
\text { coefficients) }\end{array}$ & $\begin{array}{c}\text { Test } \\
\text { de comparaison } \\
\text { des pentes } \\
\text { (Test } \\
\text { of comparison } \\
\text { of slopes) }\end{array}$ & $\begin{array}{c}\text { Test } \\
\text { de comparaison } \\
\text { des ordonnées } \\
\text { à l'origine } \\
\text { (Test } \\
\text { of comparison } \\
\text { of zcro-intercepts) }\end{array}$ \\
\hline $\begin{array}{l}\text { (I) } \\
600<\mathrm{P}<850 \mathrm{~g} \\
n=6\end{array}$ & $\begin{array}{c}\mathrm{Y}=0, \mathrm{I} 82 \mathrm{X}+28,97 \\
r=0,92(\mathrm{P}<0,01)\end{array}$ & $\mathrm{P}<0,05$ & \multirow{2}{*}{ N.S. } & \multirow{2}{*}{$\mathrm{P}<\mathrm{O}, \mathrm{O} \mathbf{I}$} \\
\hline (2) $\begin{array}{c}\mathrm{P}>850 \mathrm{~g} \\
n=15\end{array}$ & $\begin{aligned} \mathrm{Y} & =0,092 \mathrm{X}+33,05 \\
r & =0,49(\mathrm{P}<0,05)\end{aligned}$ & N.S. & & \\
\hline
\end{tabular}

I $b: \operatorname{Tr}(\mathrm{Y})$ sur $\mathrm{Ta}(\mathrm{X})$

\begin{tabular}{|c|c|c|c|}
\hline $\begin{array}{l}\text { (I) } \\
600<P<85^{\circ} \mathrm{g} \\
\\
n=\mathbf{I 0}\end{array}$ & $\begin{array}{c}\mathbf{Y}=0,377 \mathrm{X}+23,32 \\
v=0,9 \mathrm{I}(\mathrm{P}<\mathrm{0}, \mathrm{OI})\end{array}$ & $P<0,0$ I & \\
\hline (2) $\begin{array}{c}\mathrm{P}>85^{\circ} \mathrm{g} \\
n=22\end{array}$ & 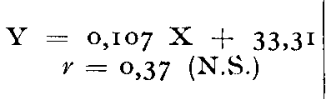 & N.S. & \\
\hline
\end{tabular}

- que, par contre, il n'y a pas, pour des températures ambiantes supérieures à $36^{\circ} \mathrm{C}$, d'augmentation brusque de la température rectale;

- que la dispersion des points expérimentaux est plus grande.

Une analyse statistique analogue à la précédente a été faite, mais cette fois-ci pour $\mathrm{Ta}$ comprise entre 23 et $40^{\circ} \mathrm{C}$. Les résultats obtenus (fig. I $b$ et tabl. I $b$ ) sont de même nature que pour la température de surface (Ts) :

- pour la classe des animaux de poids supérieur à $850 \mathrm{~g}$, la température rectale $(\mathrm{Tr})$ peut être considérée comme indépendante de Ta, lorsque celle-ci est comprise entre 23 et $40^{\circ} \mathrm{C}$;

- pour les animaux dont le poids est compris entre 600 et $850 \mathrm{~g}$, la pente de la droite de régression est significativement différente de zéro (tabl. I $b$ : test 
de 1'horizontalité de la droite) : la température rectale ( $\mathrm{Tr}$ ) augmente avec la température ambiante ( $\mathrm{Ta}$ ), et parvient au même niveau que celle des animaux de poids supérieur à $85^{\circ} \mathrm{g}$ lorsque Ta atteint $30^{\circ} \mathrm{C}$.

\section{C. - Evolution des températures corporelles en fonction du temps}

Étant donné que les températures corporelles (Ts) et (Tr) dépendent peu de la température ambiante lorsque celle-ci est inférieure à $3^{\circ}{ }^{\circ} \mathrm{C}$, du moins pour les animaux les plus lourds $\left(\mathrm{P}>85^{\circ} \mathrm{g}\right)$, on peut calculer les températures (Ts) et $(\mathrm{Tr})$ moyennes et les intervalles de confiance à un instant donné, pour les animaux de poids supérieur à 850 g. La figure 2 représente les cinétiques des moyennes de températures obtenues entre 2,5 et $60 \mathrm{mn}$ après la mise-bas.

La température superficielle ( $T s$ ) moyenne varie très peu au cours du temps.

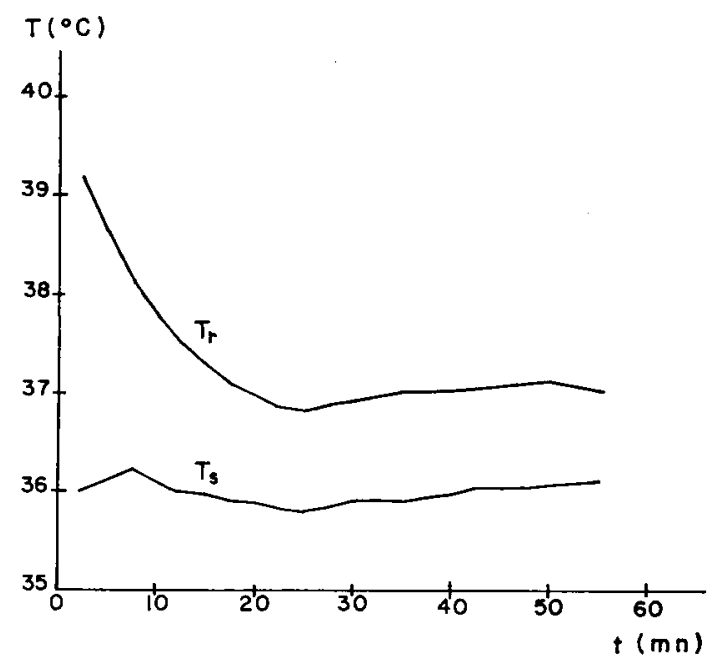

FIc. 2. - Cinétiques des températuves rectale (Tr) et superficielle (Ts) (moyennes sur I5 animaux, $\left.P>85^{\circ} g, T a<36^{\circ} \mathrm{C}\right)$

Kinetics of rectal (Tr) and surface (Ts) temperatures (means on I5 animals, $P>850 \quad g$, $\left.T a<36^{\circ} \mathrm{C}\right)$

Les intervalles de confiance à 5 p. cent sont peu importants (maximum de l'ordre de $2^{\circ} \mathrm{C}$ ).

Par contre, la température rectale $(\mathrm{Tr})$ moyenne décroît entre 2,5 et $25 \mathrm{mn}$ après la mise-bas, selon une loi de type exponentiel. Les intervalles de confiance sont plus importants que pour la température de surface (maximum de l'ordre de $\left.3^{\circ} \mathrm{C}\right)$.

\section{I). - Température critique de confort thermique du porcelet nouveau-né}

Lorsque l'animal est $\mathrm{sec}$, soit environ $25 \mathrm{mn}$ après la naissance, les pertes de chaleur par voie cutanée sont égales au produit de l'écart de température ('Ts - Ta) par le coefficient d'échange radiatif-convectif $\left(\mathrm{C}_{r}+\mathrm{C}_{c}\right)$, si on néglige la perspiration insensible (BERBIGIER, I975). 
On a donc, pour l'animal sec :

$$
\varphi_{\mathrm{M}}=\left(\mathrm{C}_{r}+\mathrm{C}_{c}\right)(\mathrm{Ts}-\mathrm{Ta})
$$

où $\varphi_{M}$ est la production de chaleur par unité de surface de peau de l'animal (W. $\mathrm{m}^{-2}$ ).

On a représenté (fig. 3) les variations de cet écart (Ts - Ta) (moyenne observée entre 25 et $60 \mathrm{mn}$ après la mise-bas) et de la thermolyse locale du dos estimée à partir de l'équation ( $\mathrm{I}$ ) en prenant un coefficient d'échange radiatif - convectif moyen $\left(\mathrm{C}_{c}+\mathrm{C}_{r}\right)=6,77 \mathrm{~W} \cdot \mathrm{m}^{-2} \cdot \mathrm{C}^{-1}$ (BERBIGIER, LE DIVIDICH et KobILINSKY, 1977), en fonction de la température ambiante (Ta).

Pour les animaux dont le poids est supérieur à $850 \mathrm{~g}$, le point anguleux obtenu pour $\mathrm{Ta}=35,5^{\circ} \mathrm{C}$ peut être considéré comme correspondant à la température critique inférieure de confort thermique.

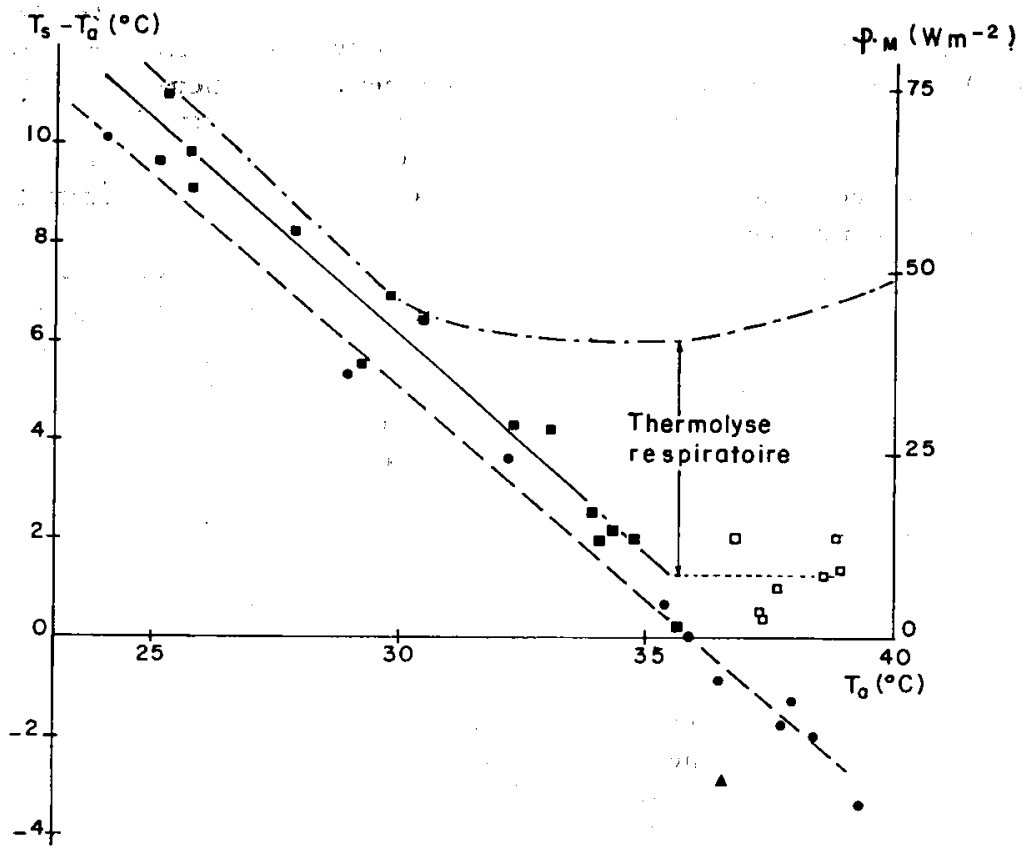

Fir. 3. - Ecart de température surjace - ambiante $(T s-T a)$ (moyenne de 25 à $60 \mathrm{mn}$ ) en fonction de la température ambiante $(T a)$.

Surface-ambient temperature difference ( $T s-T a)$ (mean from 25 to $60 \mathrm{mn}$ ) as a function of ambient temperature $(\mathrm{Ta})$.

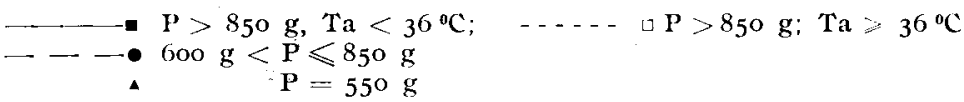

Production de chaleur $\left(\varphi_{M}\right)$ en fonction de la tompérature ambiante (Ta) en prenant $\mathrm{C}_{r}+\mathrm{C}_{c}=6,77 \mathrm{~W}^{-} \mathrm{m} \cdot{ }^{2} \cdot \mathrm{C}^{-1}$.

Heat production ( $\mathrm{TM}$ ) as a function of ambient temperature $(T a)$ assuming convective radiative exchange coefficient $\left(\mathrm{C}_{r}+\mathrm{C}_{c}\right)$ is $6,77 \mathrm{~W}-\mathrm{m} .^{2} \cdot{ }^{\circ} \mathrm{C}^{-1}$.

Production de chaleur pour un poids moyen de $1368 \mathrm{~g}$ (d'après Nicheimann, BARNICK et LYHS, 1975).

-- - Heat production for a mean weight of $1368 \mathrm{~g}$ (from Nichilmans, Barsick et Litis, 1975). 
Pour les animaux dont le poids est compris entre 600 et $850 \mathrm{~g}$ on ne retrouve pas ce point anguleux : la zone de confort semble se situer au-dessus de $39^{\circ} \mathrm{C}$.

On remarque que ('Ts - Ta) peut être nul ou mêmenégatif aux hautes températures ambiantes.

\section{IV. - Discussion et conclusion}

Parmi les facteurs ambiants influençant l'évolution des températures corporelles des animaux, on n'a retenu que la température ambiante (Ta). En effet, la température moyenne des parois ( $\mathrm{T} p$ ) est sensiblement égale à Ta; la pression partielle de vapeur d'eau de l'air à l'intérieur de l'enceinte demeure pratiquement constante, et peu différente de celle de l'air extérieur, pour des températures ambiantes ( $\mathrm{Ta}$ ) inférieures à $35^{\circ} \mathrm{C}$, ce qui signifie que la quantité d'eau évaporée est faible. Au-dessus de $35^{\circ} \mathrm{C}$, elle s'élève fortement : ceci peut s'expliquer par une augmentation de l'évaporation respiratoire des animaux (MoUNT, I968). Mais l'humidité de l'enceinte ouverte sur le milieu extérieur est fortement in fluencée par l'humidité de ce milieu, aussi l'interprétation des variations d'humidité suivant Ta doit être nuancée.

De l'analyse des résultats, on peut tirer les conclusions suivantes :

- on observe vers $35-36^{\circ} \mathrm{C}$, du moins pour les animaux dont le poids est supérieur à $850 \mathrm{~g}$, une augmentation importante de la température de surface (Ts) (fig. r $a$ ). Celle-ci est probablement due à une vasodilatation, caractéristique du confort thermique (MounT, I968). La température critique inférieure de confort thermique des porcelets nouveau-nés de poids supérieur à $850 \mathrm{~g}$, est donc d'environ $35-36^{\circ} \mathrm{C}$. On peut comparer cette valeur à celles données par d'autres auteurs poưr des animaux plus âgés : $34^{\circ} \mathrm{C}$ entre la $10^{\mathrm{e}}$ et la $\mathrm{r} 8^{\mathrm{e}}$ heure de vie (Mount, I959); 33-35 ${ }^{\circ} \mathrm{C}$ pour le premier jour (Kovacs et RAFAI, I973). I1 semble donc que la sensibilité thermique diminue dès les premières heures après la naissance, ce qui doit correspondre à la mise en place progressive de la régulation thermique;

- il existe un seuil de poids $\left(85^{\circ} \mathrm{g}\right)$ au-dessous duquel les porcelets ont des difficultés à réguler leur température de surface. En effet, les animaux dont le poids est compris entre 600 et $850 \mathrm{~g}$ ont des températures corporelles plus faibles, du moins aux températures ambiantes inférieures à $30^{\circ} \mathrm{C}$. On constate de plus, chez un animal extrêmement léger $(\mathrm{P}=550 \mathrm{~g}$ : fig. I $a$ et $b$, fig. 3$)$ que ces différences s'accentuent encore. Pour de tels animaux, la température de confort est plus élevée et n'a pas pu être atteinte au cours de l'expérience.

Les variations de Tr et Ts en fonction du poids peuvent être comparées avec les variations des coefficients d'échange cutanés (BERBIGIER et Dussuer, i977). On remarque que le seuil de poids correspondant à l'augmentation du coefficient d'échange est le même que celui qui correspond au "décrochage " de Tr et Ts. Les différences de températures corporelles pourraient n'être dues qu'à l'effet physique de la variation de poids des porcelets. On peut aussi rapprocher ceci de la diminution du taux de glucose sanguin chez les animaux de poids inférieur à $800 \mathrm{~g}$ (DUDzus et UECKER, I976). Cependant, la portée de ce résultat est limitée par le fait que les animaux légers représentent moins de 5 p. cent des effectifs des portées, et ont le plus souvent un avenir zootechnique assez compromis : 
animaux peu compétitifs à la têtée, faible vitesse de croissance consécutive à une ingestion limitée d'aliments.

Mais le point le plus intéressant est sans doute que la régulation initiale de température ( $I^{r e}$ heure de vie) se situe essentiellement au niveau superficiel, et qu'elle a lieu avant la stabilisation de la température rectale, qui résulte des échanges thermiques entre la surface et les parties centrales. En effet, la température de surface ( $\mathrm{T}$ s) ne dépend, sauf pour les animaux les plus légers $(600<\mathrm{P} \leqslant 850 \mathrm{~g})$, ni de la température ambiante (entre 23 et $3^{\circ}{ }^{\circ} \mathrm{C}:$ fig. I $a$ ), ni du temps (à partir de $2,5 \mathrm{mn}$ après la mise-bas : fig. 2). Par contre, la température rectale $(\mathrm{Tr}$ ) décroît selon une loi de type exponentiel (fig. 2) caractéristique du refroidissement d'un solide inerte (pas de régulation centrale). I a régulation superficielle a probablement une origine musculaire (frisson : BuTchBAKER et SHANkLIN, I965).

Une représentation plus classique du confort thermique est donnée par la figure 3(qui ne traduit que la thermolyse cutanée dorsale). Celle-ci peut s'inverser aux hautes températures ambiantes (Ta $>\mathrm{Ts}$ ) : la thermolyse doit se faire alors par évaporation respiratoire.

Les valeurs de la thermolyse cutanée obtenues pour Ta compris entre 23 et $40^{\circ} \mathrm{C}$ sont comparables à celles de la thermogenèse données par NichermanN, BARNICK et LYHS (I975, fig. 3), sachant que la méthode utilisée ici ne rend pas compte des échanges respiratoires. I1 semble donc que la valeur de $6,77 \mathrm{~W} . \mathrm{m}^{-2} \cdot{ }^{0} \mathrm{C}^{-1}$ soit une bonne estimation du coefficient d'échange radiatif-convectif $\left(\mathrm{C}_{c}+\mathrm{C}_{r}\right)$ moyen, et que la température de surface du dos représente bien la température de surface moyenne pondérée par les coefficients d'échange correspondant aux différentes parties du corps de l'animal. Joyce, BLAXTer et PARK (I 966) trouvent d'ailleurs, sur le mouton, un coefficient d'échange radiatif-convectif similaire (de 6,25 à $7,15 \mathrm{~W} \cdot \mathrm{m}^{-2} \cdot{ }^{0} \mathrm{C}^{-1}$ ) sensiblement inférieur à la valeur théorique $\left(8,35\right.$ W.m. $\mathrm{m}^{-2} \cdot{ }^{0} \mathrm{C}^{-1}$, Winslow, GaGge et Herrington, I940).

L'aspect de la courbe de la figure 3 est le même que celui des courbes de confort thermique (production de chaleur en fonction de la température ambiante) obtenues chez le porc plus âgé (KIEIBER, I96I), bien que la température rectale ne soit pas constante chez le nouveau-né pendant la première heure de vie. Des régulations de natures différentes (centrale dans un cas, superficielle dans l'autre), aboutissent cependant au même type de variation des échanges thermiques en fonction des conditions ambiantes.

En conclusion, les résultats présents montrent que, chez le nouveau-né, les mécanismes de régulation thermique superficielle sont primordiaux. Cette régulation s'exerce de manière à maintenir constante la température de surface de la peau, quelle que soit la température ambiante (entre 23 et $36^{\circ} \mathrm{C}$ ) et le temps écoulé depuis la mise-bas (jusqu'à une heure). La température centrale par contre est peu régulée. Ce mécanisme est très différent de celui que l'on constate lorsque l'homéothermie est bien établie. Cependant la forme des courbes de confort thermique (production de chaleur en fonction de Ta) est la même. La température critique inférieure de confort thermique semble être de l'ordre de $35-36^{\circ} \mathrm{C}$, pour les porcelets nouveaux-nés ayant un poids supérieur à $850 \mathrm{~g}$. 


\section{Summary}

\section{Influence of the environmental temperature on body temperature kinetics and thermal comfort in neonate piglets}

The skin temperature (Ts) measured by infra-red radiothermometry as well as the rectaI temperature ( $\mathrm{Tr}$ ) of 33 Large White piglets were recorded continuously during the animal's first hour of life. The variations in the body temperature and the evolution of heat losses in the piglets were measured at environmental temperatures (Ta) ranging between 23 and $40^{\circ} \mathrm{C}$.

During the ist hour of life, the regulation of the temperature seems mainly to be located at the body surface, except in the lightest animals $(P \leqslant 850 \mathrm{~g})$ which have difficulties in maintaining a constant skin temperature (Ts). This regulation is most likely of muscular origin (shivering).

The mechanism of surface temperature regulation in the neonate is very different from that of the older homeothermal pig (central regulation). However the shape of the thermal comfort curves (heat production according to Ta) is the same.

The lower critical temperature of thermal comfort is about $35^{-3} 6^{\circ} \mathrm{C}$ for animals weighing more than $850 \mathrm{~g}$. This temperature can be accurately determined by measuring the skin temperature (Ts). From a temperature of $35-36^{\circ} \mathrm{C}$, a large increase in $\mathrm{T}$, characteristics for thermal comfort, was observed.

\section{Références bibliographiques}

Berbigier P., I975. Sur la mesure des échanges de chaleur au niveau de la peau des porcs élevés sous abri par la méthode du bilan d'énergie. Ann. Zootech, 24, 413-422.

Berbigrer P., Dussuer. A., 1977. Étude des cinétiques de température et du confort thermique du porcelet pendant l'heure suivant la mise-bas. Strasbourg : Rencontre S.F.T.Energétique et thermique de l'homme dans son environnement.

BERBIGIFR P., LE DIVIDICH J., KOBIIINSKY J., I978. Étude des cinétiques des températures rectale et superficielle chez les porcelets à la mise-bas : porcelets nés en maternité à ambiance constante. Ann. Zootech. (sous presse).

Butchbaker A. F., ShankLiN M. D., 1965. Development of homeothermic regulation in young pigs. Trans. Am. Soc. Agr. Engrs, 9, 481-485.

Chiapale J. P., 1970. Emploi de 1'anémomètre à fil chaud dans les mesures microclimatiques. In "Techniques d'étude des facteurs physiques de la biosphère ", I.N.R.A., Paris, 409-42I.

DE la Porte des Vaux H., Aumaitre A., I967. L'influence du milieu thermique sur l'évolution de la température rectale et de la glycémie chez le porcelet nouveau-né. Ann. Zootech., 16, $235-245$.

DUDzUs V. M., UECKER E., 1976. Studies into carbohydrate metabolism in new-born piglets (Allemand). Mh. Vet. Med., 31, gro-9r3.

Erneir H., MCCANCE R. A., 1965. The effect of environmental temperature in the composition and carbohydrate metabolism of the newborn pig. J. Physiol., Lond., 179, 278-284.

ENGirish P., 1976. Sow special. Pig liarming, 24, 65-67-72.

JOYCE J. P., BLAXTER K. L., PARK C., 1966. The effects of natural out-door environments on the energy requirements of sheep. Res. Vet. Sci., 7, 342-359.

KeRN KAMP H. C. H., I965. Birth and death statistics on pig of preweaning age. J. Am. liet. Med. Assoc., 146, 337-340.

KLEIber M., I96r. The fire of life. John Wiley and Sons, New-York, Lond., 454 p.

Kovacs F., RAFAI P., I973. Investigation on the metabolism of new-born and young piglets (Hongrois) Magyar Ao. Lapja, 28, I8?-I87.

Kovacs F., RaFai P., Sal, health and production of pigs. $I$. The effect of the temperature on the organism of newborn and young piglets (Hongrois). Magyar Ao. Lapja, 22, 449-454.

Movnt L. E., 1959. The metabolic rate of the new-born pig in relation to environmental temperature and to age. J. Physiol., Lond., 147, 333-345. 
Mount L. E., x968. The climatic physiology of the pig. Edward Arnold Ltt., Lond., $27 \mathrm{r}$ p. Nicher,mann M., Barnick H. G., Lyirs I., r975. Studies into heat regulation of new born swine. Ist communication: heat production (Allemand). Mh. Vet. Med., 30, 348-353.

Nielseis N. C., Christensen K., Bille, N., IARSen J. L., 1974. Preweaning mortality in. pigs. I. Herd investigations. Nord. Vet. Med., 26, I37-I50.

PERrier A., I970. Mesure des températures de surface par radiométrie infra-rouge. In "Techniques d'étude des facteurs physiques de la biosphère ", I.N.R.A., Paris, I69-I78.

Stombaugh D. P., Roller W. L., Adams T., Teagul H. S., 1973. Temperature regulation in neonatal piglets during mild cold and severe heat stress. Am.J. Physiol, 225, i 192-1 198. Widpowson E. M., I950. Chemical composition of newly born mammals. Nature, Lond., 166, 626-628.

Winstow C. E. A., GagGe A. P., Herrington L. P., I940. Heat exchange and regulation in radiant environments above and below air temperature. Am. J. Physiol., 131, 79-92. 